\title{
Study of Uplink Interference in UMTS Network: ASIACELL Company, Iraq
}

\author{
Bakhtiar Ali Karim \\ Communications Engineering \\ Technical College of Engineering \\ Sulaimani Polytechnic University \\ Sulaimani, Iraq \\ Bakhtiar.ali@spu.edu.iq
}

\author{
Bzhar Rahaman Othmaan \\ Communications Engineering \\ Technical College of Engineering \\ Sulaimani Polytechnic University \\ Sulaimani, Iraq \\ bzhar.rahman@spu.edu.iq
}

Article Info
Volume 5-Issue 1-June
2020
DOI:
10.24017/science. 2020.1 .7

Article history:

Received: 04 April 2020 Accepted: 07 June 2020

\section{Keywords:}

RTWP,

UMTS,

Interference,

$3 \mathrm{G}$.

\begin{abstract}
Universal Mobile Telecommunications System (UMTS), is the third generation (3G) of mobile communication which is based on the wideband code division multiple access (W-CDMA) radio access to provide bandwidth and spectral efficiency. Interference in $3 G$ system is significantly lower compared to the preceded generations. However, it does not mean $3 G$ is free from the issues associated with interferences, such as low signal quality and call drop. The interference level in UMTS can be measured by using the well-known parameter Received Total Wideband Power (RTWP). This parameter is affected by many factors such as number of the users connected to the system, combining second generation (2G) and $3 G$ frequencies within the same geographical area, geographical causes (difference of Altitude), and hardware impairment. In this paper we intensively study how these factors affect the uplink interference level (i.e. RTWP value) in $3 G$ system used by a particular telecommunications company, Asiacell company, Iraq. The obtained data shows that call drop is the most serious issue raised due to high value of RTWP in $3 G$ system. We demonstrate that system enhancement, in terms of lower RTWP level, are obtained by adding second carrier to the sites, separating $2 G$ band from $3 G$ band using special filter, and optimizing the hardware components.
\end{abstract}

Copyright $(2020$ Kurdistan Journal of Applied Research. 


\section{INTRODUCTION}

Telecommunication sectors and the Internet are integrated quickly due to the rapid developments made in communications technologies. Merging these two sectors and the needs for "All IP" services realize $3 \mathrm{G}$ system to fulfill the requirements [1]. The European standard for 3G known as universal mobile telecommunication systems (UMTS) introduced by two standard bodies ETSI in Europe and ARJB in Japan. UMTS is considered as an evolution in terms of increasing communications speed and service types. UMTS is an interference limited system that the signal of each user introduces severe interferences for others if there is a lack of orthogonality among their codes [2].

The growing demands for high data connectivity and wireless Internet access are addressed in UMTS which guarantees up to $384 \mathrm{kbps}$ and 2 Mbps data rates for moving users and stationary users respectively. Compared to the second generation (2G), UMTS are introduced not only to guarantee higher speed, but also variety new services to the mobile users including access to broadcast TV, web browsing, email, and fax in addition to global roaming capability [3]. 3G system built based on the GSM standard because market was dominated by GSM technology before the invention of UMTS [4].

Different studies have been conducted in literature regarding uplink interference in 3G system [5-10]. Other-cell interferences under different model of propagations are presented in [5-6]. The authors assumed that fixed and equal number of users, who are evenly spread, exist at each cell. The focuses of the authors in [5] is on the first moment, while [6] investigates the second moment of other-cell interferences. In [7] various analytical methods are developed to characterize the other-cell interference in UMTS network. Authors of [8] introduced an effective interference management method for $3 \mathrm{G}$ network integrated with femtocells, in which adaptive attenuation technique at the femtocells is proposed to mitigate uplink interferences. According to their results not only coverage, but also capacity enhancement can be achieved when this technique is applied to a network combined of $3 \mathrm{G}$ and femtocells. Authors in [9] proposed an uplink interference cancelation (IC) scheme known as Post Decoding IC. They stated that the high quality channel estimation is one of the primary requirements to provide efficient IC technique in W-CDMA/HSPA receivers. Path-gain based uplink interference estimation technique is studied in [10]. The method is based on the combination between input and the average estimated interference power, which is obtained from the knowledge of the path gain matrix, over specific period and the technique works well under various situations.

None of the mentioned works stated the reasons and the consequences of uplink interferences on a particular system, instead they analyzed new techniques in general. Thus, to the best of the authors' knowledge, this type of work has not been studied in this form in literature. Therefore, this work is different from all the works done before in terms of the way the research is conducted (we practically collected the data for a specific UMTS system used by Asiacell company in Sulaimani city, Iraq) and the content of the obtained results.

The rest of the paper is organized as follows: Section two explains interference clarifications, particularly inter-cell and intra-cell interferences, in UMTS network. In section three, the consequences of interferences and the factors that cause increasing RTWP level, in UMTS system used by Asiacell company are analyzed, and the obtained results are presented and comprehensively discussed. The final section summarizes the main points of the paper.

\section{INTERFERENCE IN UMTS SYSTEM}

\subsection{Architecture of UMTS network}

Three main parts mentioned below form the general architecture of UMTS system: 


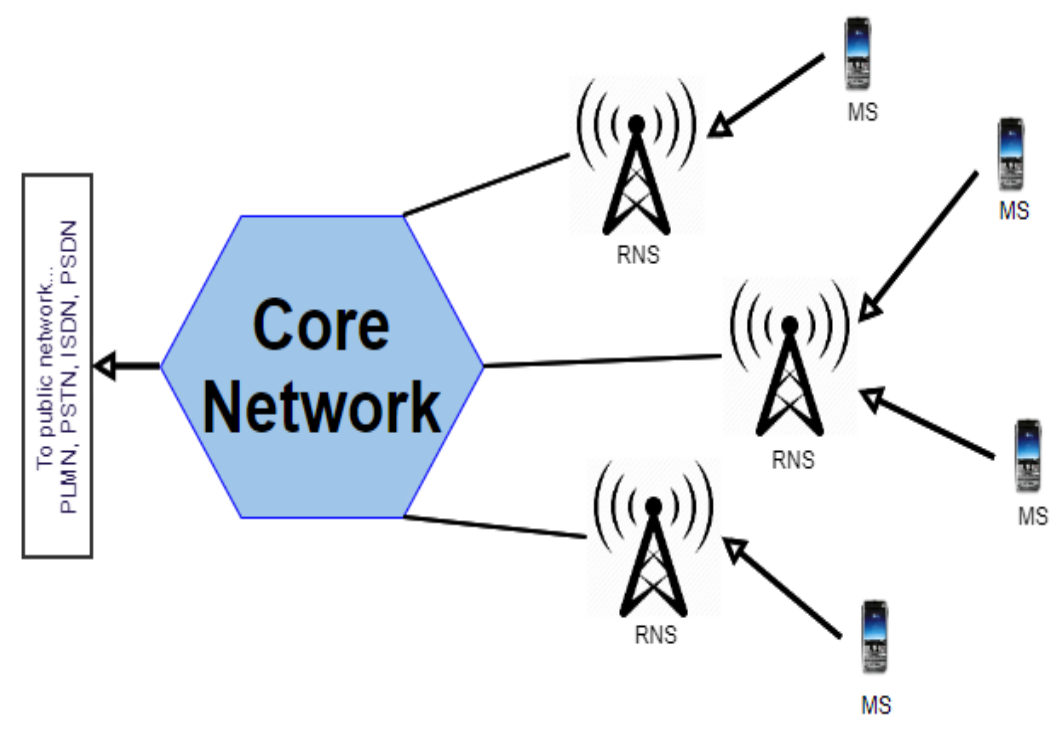

Figure 1: UMTS network architecture overview [11]

\subsubsection{User Equipment (UE)}

EU is the name of the device directly used by the end user and it is the corresponding name of mobile station (MS) used in 2G.

\subsubsection{Radio Network Subsystem (RNS)}

RNS in 3G called UMTS Terrestrial Radio Access Network (UTRAN), it is the equivalent part of the Base Station Subsystem (BSS) in GSM network. RNS compromises of base stations and radio network controller (RNC) which provides and manages the air interface between UE and the core network.

\subsubsection{Core Network (CN)}

The $\mathrm{CN}$ is the main part of the UMTS network which provides the services to the end users. Circuit switched and packet switched communication services are supported by UMTS CN. CN has the functionality done by Network Switching Subsystem (NSS) in GSM. Different type of networks, such as public switching telephone network PSTN, are interfaced to the CN to support different communication protocols.

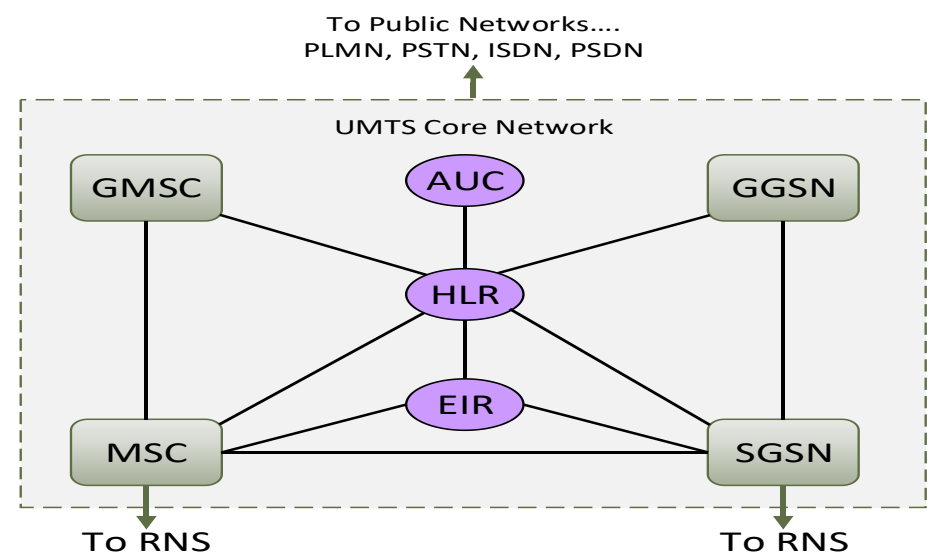

Figure 2: UMTS Core Network [11] 


\subsection{Interferences}

Interference is a phenomenon that occurs in wire and wireless communication systems when two or more signals having the same characteristics pass through the shared medium. The result of this phenomenon is the corruption of the wanted signals by undesired signals. This issue has been the main barrier in front of communication-system designers to optimize the system.

\subsubsection{Interference classification}

In cellular network generally interference has different forms [12]:

- Radio Frequency Interference (RFI): This is a type of interference, also called electromagnetic interference (EMI), is introduced in a wireless system when a nearby external RF source, such as TV or radio transmitter, operates on the same frequency. To avoid the wireless systems from this type of interference, it is crucial to have a sufficient geographical space between the RF sources operating on the same frequency bands.

- Electrical Interference (EL): This category of interference is generated by electrical equipment, such as digital devices, computers, lighting systems. This form of interference is not intentional since nobody take advantages out of it.

- Intermodulation (IM): This class of interference is created by the system itself rather than external sources. The IM is generated in a nonlinear system due to unwanted combination of different type of signals which produces new and undesired frequencies. This newly generated frequencies will negatively affect the adjacent systems. Generally, nonlinear power amplifiers are considered as an active source of IM.

\subsubsection{Interference in UMTS network}

Due to using CDMA technique, interference in $3 \mathrm{G}$ is lower than that in the $2 \mathrm{G}$ network. However, it does not mean the interference in UMTS system is negligible. Since $3 \mathrm{G}$ is an interference-limited system, interference analysis is the primary criteria to evaluate the performance of UMTS network. Generally, the total interference within a particular system composed of intra-cell and inter-cell interferences which are introduced at each uplink and downlink scenarios [13] [14].

\section{- Intra-cell interference}

In the uplink, all the other mobile users, who are in the coverage region of a single base station (BS), cause intra-cell interference to the connected user. Whereas, in the downlink scenario, the home BS introduces this type of interference to a user. This is due to the absence of orthogonality, which is caused by the multipath effect, among the served mobile users [15].

\section{- Inter-cell interference}

In the uplink scenario, inter-cell inference is the total of the signals received from all users located in a cell other than the cell the mobile user connected to. While in the downlink, this type of interference is the result of receiving signals from surrounding BSs [15]. Both type of interferences in uplink and downlink scenarios are illustrated in the following figures. 


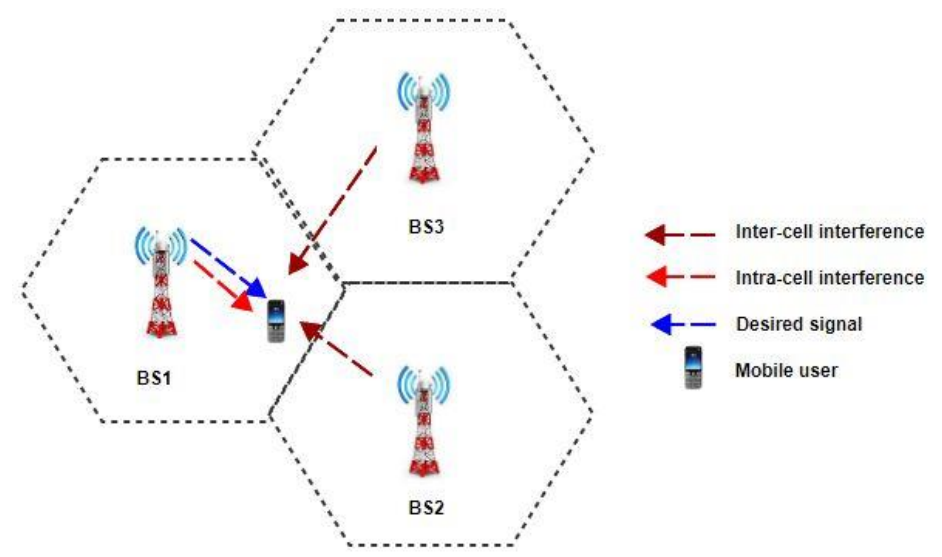

Figure 3: Inter-cell and Intra-cell interferences in Downlink scenario

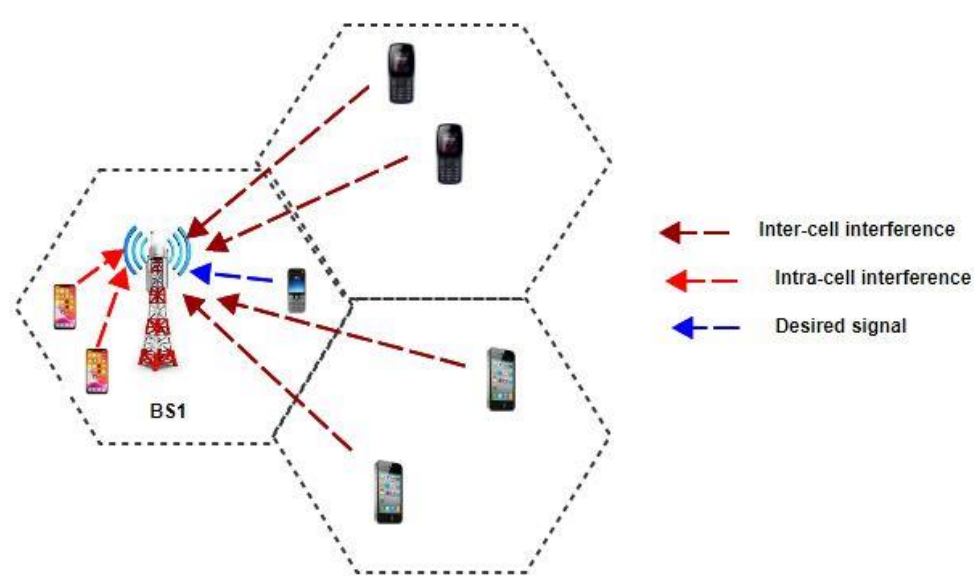

Figure 4: Inter-cell and Intra-cell interferences in Uplink scenario

\subsubsection{Internal and External interferences.}

Interference can also be categorized as internal and external interferences:

\section{- Internal interferences}

This interference occurs due to fault in the equipment. The following factors are the main causes for the internal interference [16]:

- If the antenna connector is not screwed properly (too loose or too tight)

$\circ$ If there are too much bending or damages in the antenna feeder.

$\circ$ If power amplifiers are spurious.

The following characteristics are observed from this class of interference [16]:

- RTWP value for the main path and diversity path are different, whereas it is the same for different frequencies.

- The data traffic and the interference are correlated. In the other words, with the absence of traffic there is a low probability of having interference in the system.

\section{- External interferences}

Interference could be happening due to some external sources that use frequencies close to the bands used by UMTS network operators. The external sources can be phone jammers or any microwave transmission devices or illegal use of UMTS band by other operators. This class of interference has the following characteristics [12]:

- In normal cases, RTWP value is the same for both main path and diversity path

- In cellular systems, cells with geographical proximity are also affected by this type of interference. 
The focus of our paper is on the uplink interference since it is easier to measure and collecting data from base transceiver stations (BTSs) is far less complex compared to the data collection at the UE (for downlink interference data need to be collected from UE). Additionally, there is no a reliable way to measure RTWP level at UE from the radio access network control center at Asiacell company, whereas reliable data from the BTSs can be achieved easily.

In this paper, in addition to the consequences of high RTWP value, we analysis the factors that affect the RTWP level which is the basic criteria to measure the uplink interferences in the UMTS system.

\section{RESULTS AND DISCUSSION}

\subsection{RTWP Values}

One of the more realistic measures to obtain the total noise level within a particular cell of 3G/UMTS system is RTWP value. This criterion is related to the uplink interference which is the main reason for call drop in UMTS network.

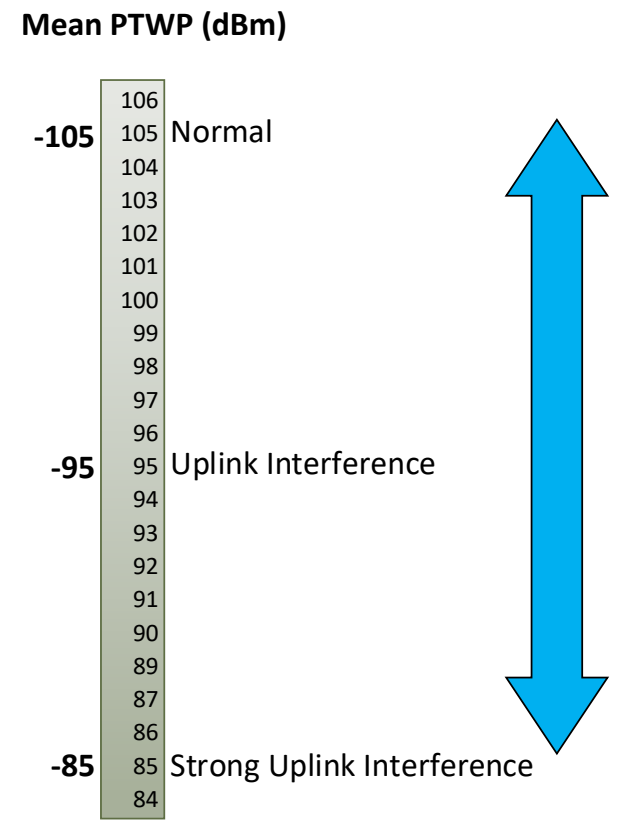

Figure 5: RTWP ranges

As shown in the figure 5, RTWP around $-105 \mathrm{dBm}$ is a normal situation and denotes that the uplink interference is under control. RTWP value close to $-95 \mathrm{dBm}$ indicates that there is an acceptable level of uplink interference. Whereas, $-85 \mathrm{dBm}$ shows a strong interference in the system that needs to be resolved since it causes problem for the desired signals.

The measures of RTWP value can be low, medium, and high. The maximum and minimum values, however, are used only as references since these values may be caused by the high access level in the network. Therefore, the value that we focus on in this paper and has most accurate information is the mean RTWP value. To illustrate that we have measured RTWP for two different sites which has different values as shown in figure 6. 


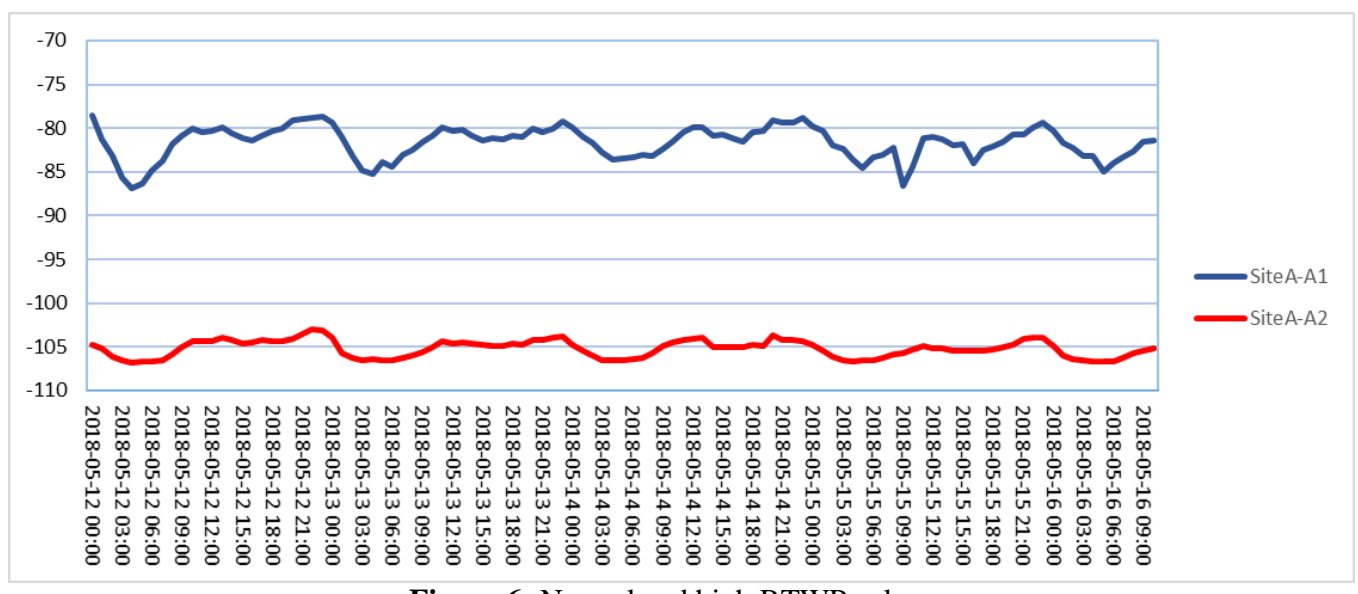

Figure 6: Normal and high RTWP values

According to the result in figure 6, siteA-A2 has lower RTWP while siteA-A1 has higher RTWP. Thus, uplink interference in siteA-A2 is comparatively less than that in siteA-A1. This could be due to one of the reasons explained in the following sections.

\subsection{The problem introduced due to high value of RTWP}

One of the main issues introduced due to high RTWP value and degrades quality of services (QoS) is the call drop. To illustrate that we have measured mean RTWP and call drop rate within a particular cell in Asiacell company as shown in the figure 7.

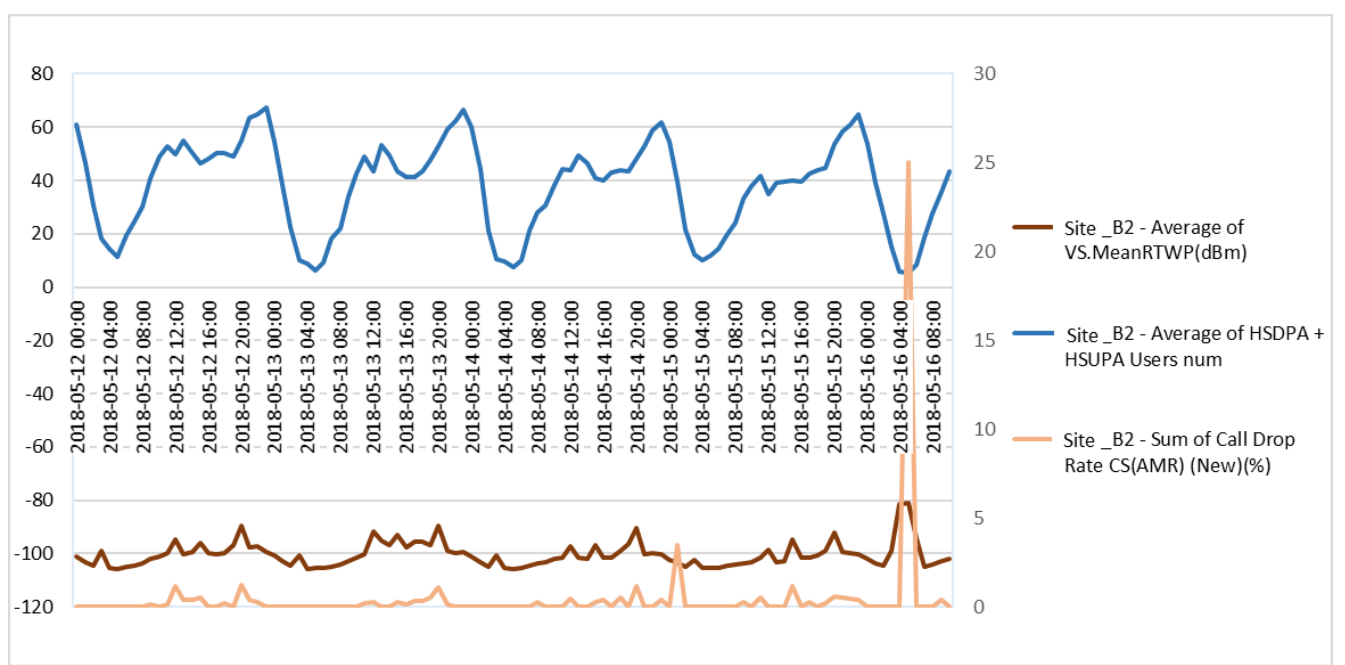

Figure 7: Call drop rate due to high RTWP level

The figure clearly shows that RTWP values directly affect call drop rate. Call drop is close to 2 when RTWP was near $-100 \mathrm{dBm}$. However, when RTWP reaches $-80 \mathrm{dBm}$, highest call drop rate has been recorded which is nearly 25 calls. The figure also shows that with highest call drop rate the average number of the serving users fall to the lowest number (from over 60 users to under 20 users).

\subsection{The factors of increasing RTWP in Asiacell company:}

In UMTS system, the value of RTWP may be affected by several reasons including the number of users connected to the system, using part of $2 \mathrm{G}$ frequency in $3 \mathrm{G}$, geographical reasons, and hardware impairment. 


\subsubsection{Number of users}

Increasing number of subscribers results high uplink interference, thus high value of RTWP. To confirm that, we have calculated mean RTWP within different times while the number of the connected users, to the system, vary as shown in figure below.

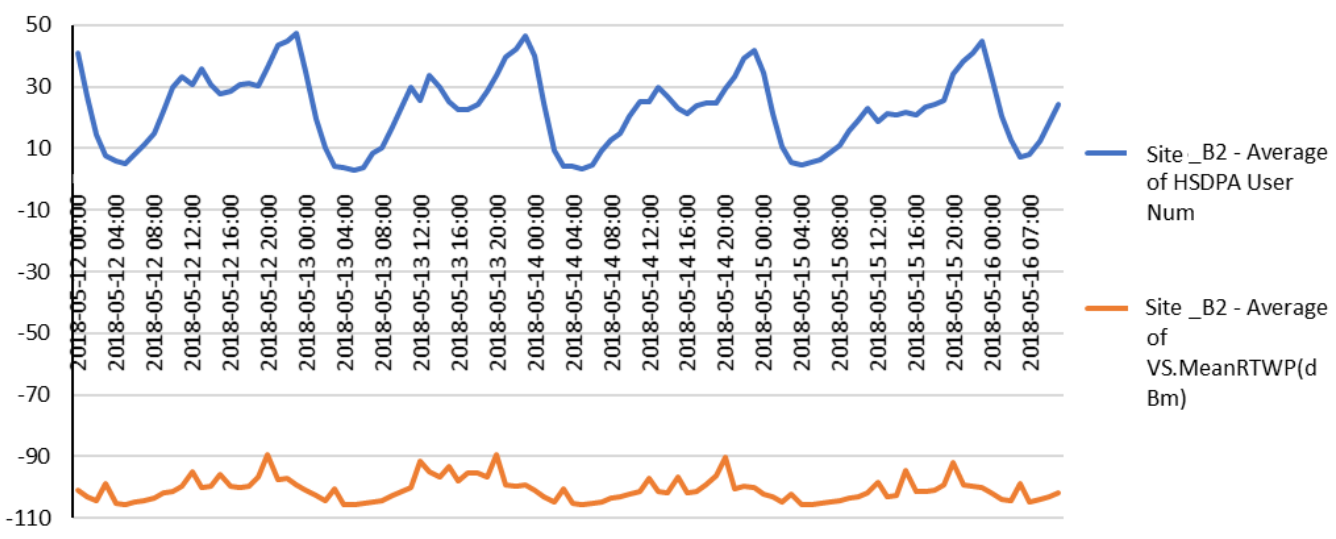

Figure 8: Increasing RTWP due to increasing number of users.

From the figure 8, it can be observed that when the number of users is minimum (about 3 users per site), lowest value of RTWP has been recorded which is under $-100 \mathrm{dBm}$. Whereas, the number of users reaches just under 50 , the RTWP value jumped to $-90 \mathrm{dBm}$. Therefore, number of users has direct effect on the uplink interference in $3 \mathrm{G}$ system.

One way to solve this problem is adding second carrier to the cell which has high RTWP. Through this way, the connected users will be divided into two frequency bands rather than one. Consequently, the interference between the users will be decreased, thus results lower RTWP value. We have investigated this approach lower and stable RTWP values has been recorded.

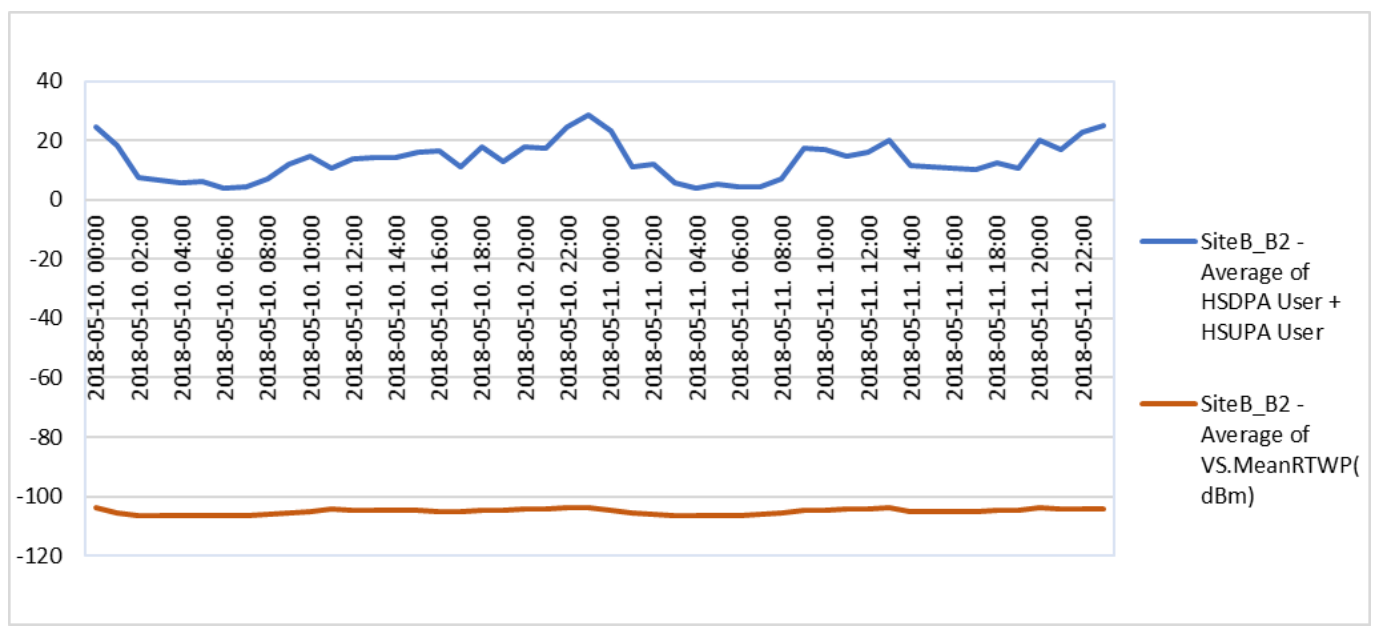

Figure 9: RTWP reduction due to adding second carrier.

As shown in the figure 9 due to adding second carrier, RTWP stayed under $-100 \mathrm{dBm}$ for the entire period even though number of users fluctuate in this sites. This is a clear indication that RTWP can be controlled by using extra carrier in the same site. However, when applying this method, the spectrum-limitation problem needs to be considered. 


\subsubsection{Using part of $2 G$ frequency bands for $3 G$}

In Asiacell company, part of the $2 \mathrm{G}$ spectrum, around $900 \mathrm{MHz}$, are re-used in the $3 \mathrm{G}$ system. In some areas, for special reasons, $3 \mathrm{G}$ spectrum are also re-used for $2 \mathrm{G}$ system at the same site or neighboring sites. The interferences caused by combination of $2 \mathrm{G}$ and $3 \mathrm{G}$ bands result higher RTWP values as shown in figure below.

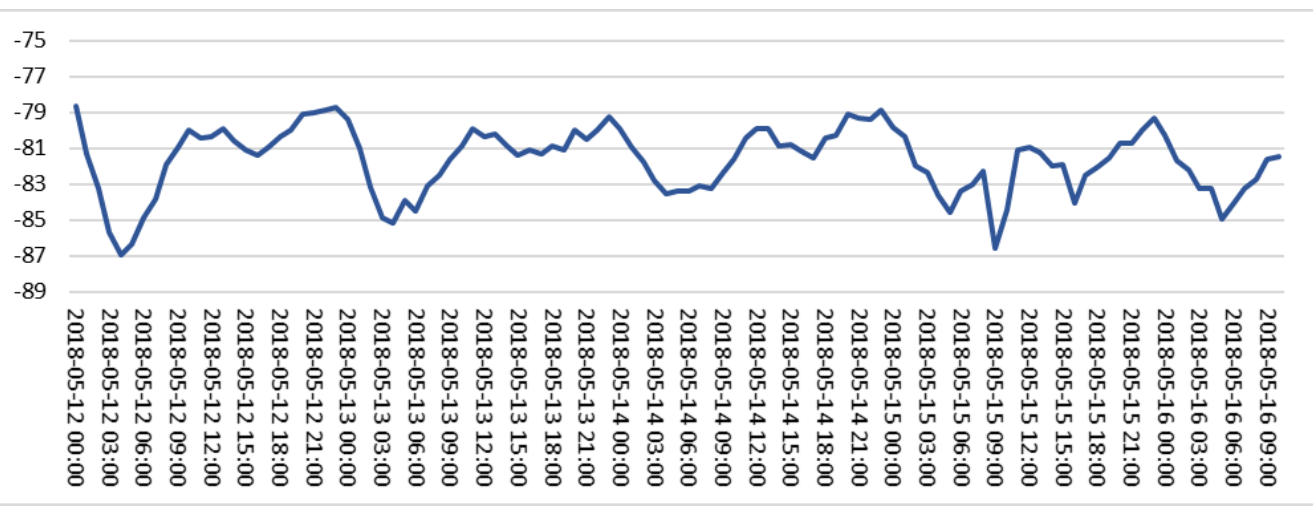

Figure 10: High RTWP values due to re-using $2 \mathrm{G}$ spectrum within $3 \mathrm{G}$ sites

From the figure it can be seen that RTWP is between $-79 \mathrm{dBm}$ and $-87 \mathrm{dBm}$. This is unacceptable range of uplink interference and significantly affect the QoS.

To address the problem of $2 \mathrm{G}$ and $3 \mathrm{G}$ bands interferences, it is required to add a special filter into the $3 \mathrm{G}$ sites to filter out all frequency bands except $3 \mathrm{G}$ band. To prove that we have compared the RTWP values of two sites, one with filter and another without filter. As shown in the figure 11, the site which has no filter experiences higher value of RTWP compared to the one with filter.

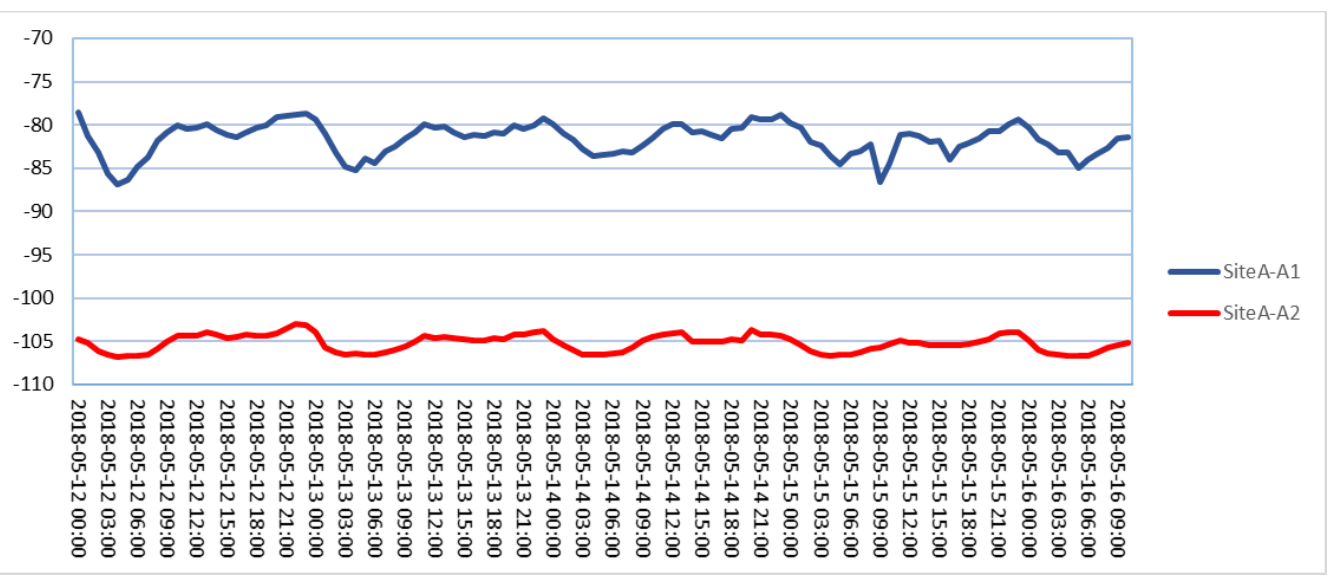

Figure 11: RTWP reduction due to using filter in $3 \mathrm{G}$ sites to reject $2 \mathrm{G}$ bands (SiteA-A1 without filter, SiteA-A2 with filter)

The figure depicts that the RTWP was kept lower, about $-105 \mathrm{dBm}$ in average, in SiteA-A2 due to filtering out $2 \mathrm{G}$ spectrum. Whereas, RTWP stayed high, approximately $-83 \mathrm{dBm}$ in average, in SiteA-A1 because of not using filters to separate $2 \mathrm{G}$ band from $3 \mathrm{G}$ bands.

It is noticeable that figure 6 and figure 11 look similar but they are presented for different purposes. The former one aims to show that RTWP level is vary from one site to another, 
whereas the later one confirms the fact that RTWP value can be minimized using special filter in the sites.

\subsubsection{Geographical causes (difference of Altitude)}

In this research we focused on the specific area, Sulaimani city, Iraq. Naturally, the city has significant differences in altitude from one place to another. Such that the east part of the city has higher altitude compared to the west part as shown in the figure 12 . Therefore, if the problem is not solved through tilting the signals of the cells located east of the city propagate towards the cells located west of the city. Consequently, due to this issue the cells at the west part of the city experience higher RTWP thus higher uplink interferences.

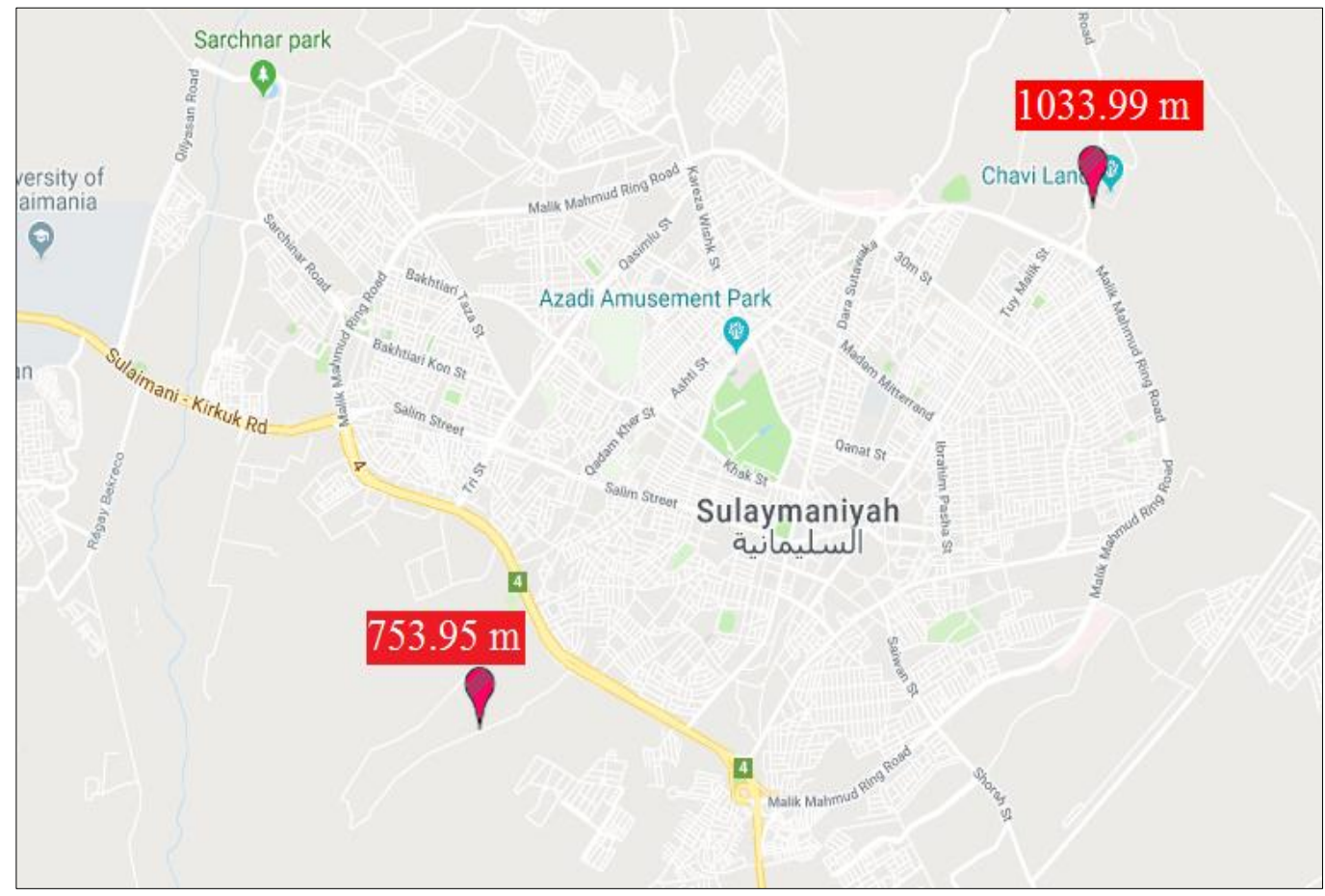

Figure 12: Difference Altitude between east and west of Sulaimani city

Asiacell company has tackled this problem by arranging the azimuth and tilt of the antennas that is suitable with the geographical nature of the city. Therefore, no data has been recorded since this problem does not exist in the Asiacell company.

\subsubsection{Hardware causes}

During our research in Asiacell company, we have also observed that malfunction of some hardware equipment, such as RRU, filters, cables, and jumpers, causes problems for signal transmission and reception, and this results increasing RTWP. 


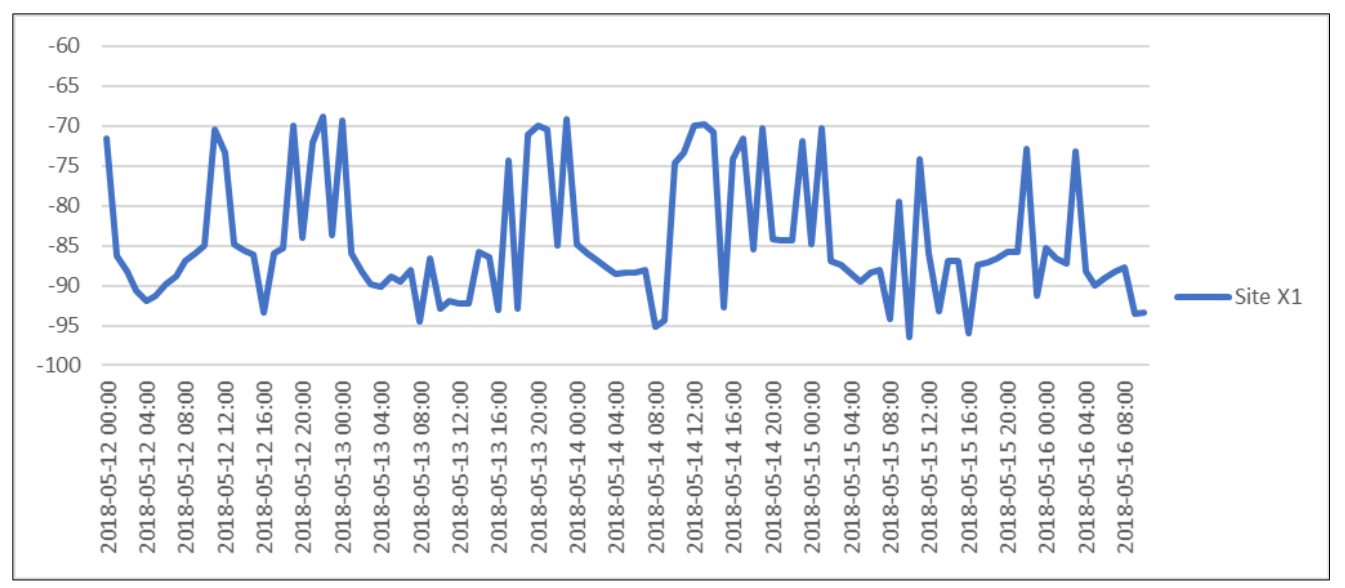

Figure 13: High RTWP due to hardware impairments

The result indicates that the RTWP value is high (higher than normal range) since the Site X1 has problem in its hardware components.

Due to time constraint and other technical reasons, we would not be able to measure the data of Site X1 after repairing the hardware impairments, meanwhile the hardware faults at Site X2 had already been solved. Therefore, the data was collected at Site X2, not Site X1, to indicate the effect of hardware cause on RTWP level.

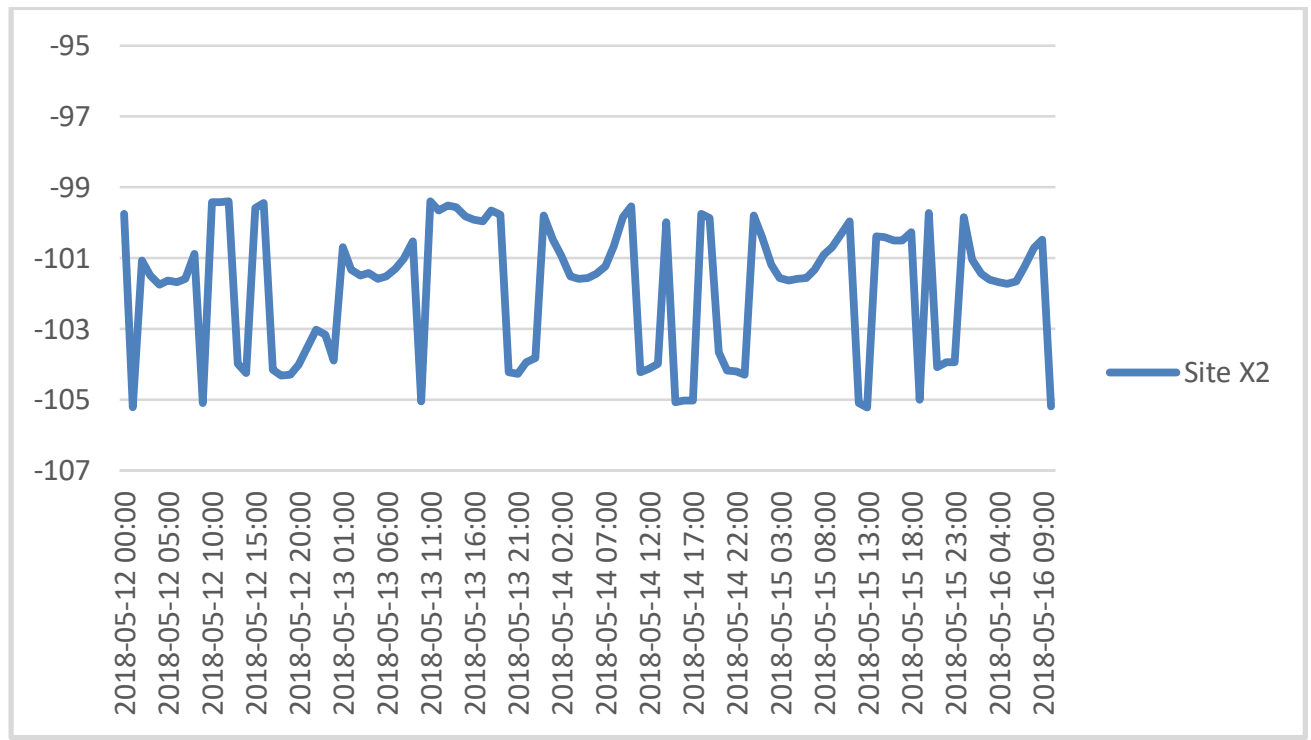

Figure 14: Lowering RTWP after repairing hardware equipment

This result indicates that the RTWP value retuned into its normal range as the malfunction of the hardware components has been resolved.

\section{CONCLUSION}

In this paper interference in $3 \mathrm{G}$ system particularly uplink interference in UMTS network has been studied. The primary focus of the paper has been on the factors that influence RTWP value, thus the uplink interference, in the UMTS system used by Asiacell company. It has been proven 
that high RTWP value results higher call drop rate, therefore, reducing number of the serving users within a particular cell. The presented results have shown that RTWP level is proportional to the number of connected users to a dedicated cell. High RTWP level has been recorded as the number of user increase. This problem, however, can be addressed by adding second carrier to the cell. Re-using GSM frequency bands for UMTS system also causes high RTWP value. Using filter in UMTS sites for receiving desired signals has been shown as a solution for such problem. Geographical causes and hardware impairments also play a noticeable role at determining RTWP level in UMTS network.

\section{REFERENCE}

[1] C. Kappler, "UMTS networks and beyond", John Wiley \& Sons; 2009

[2] G. Paschos, et al., "The impact of intermodulation interference in superimposed 2G and 3G wireless networks and optimization issues of the provided QoS", The impact of intermodulation interference in superimposed $2 \mathrm{G}$ and gr/Archive/Papers. 2003.

[3] M. Kyum, et al., "Performance analysis of umts cellular network using sectorization based on capacity and coverage", International Journal of Advanced Computer Science and Applications, 2(6), pp. 98-104, 2011.

[4] G. He, Overview of UMTS. Telecommunication Software and Multimedia Laboratory, Helsinki University of Technology. 2001.

[5] K. Gilhousen, et al., "On the capacity of a cellular CDMA system", IEEE Transactions on Vehicular Technology, 40(2), pp. 303-312, 1991.

[6] K. Kim, "CDMA cellular engineering issues", IEEE Transactions on Vehicular Technology, 42(3), pp. 345350,1993

[7] T. Liu and E. David, "Interference and outage probability evaluation in UMTS network capacity planning", European transactions on telecommunications, pp. 651-660, 2007.

[8] M. Yavuz, et al., "Interference management and performance analysis of UMTS/HSPA+ femtocells", IEEE Communications Magazine, 47(9), pp. 102-109, 2009.

[9] S. Sambhwani, W. Zhang, and W. Zeng, "Uplink interference cancellation in HSPA: principles and practice". Qualcomm Inc. 2009.

[10] EG. Lundin, "Uplink admission control based on estimated interference in WCDMA systems", Master's Thesis completed at Communications Systems, Linkopings Universitet, Sweden. 2001.

[11] H. Kaaranen, et al., "UMTS networks: architecture, mobility and services", John Wiley \& Sons; 2005.

[12] A.Springer, et al., "Effects of Nonlinear Amplifier Distortion on UMTS Transmitter System Performance," Paris, In European Conference on Wireless Technologies, 2000.

[13] I. Iskandar, I. Setyawan, and H. Nuraini, "Inter-cell Interference Management Technique for Multi-Cell LTEA Network", International Journal of Electrical and Computer Engineering; 7(5), pp. 2696-2705, 2017.

[14] Y.A. Adediran, H. Lasisi, and OB. Okedere, "Interference management techniques in cellular networks: A review", Cogent Engineering, 4(1):1294133, 2017.

[15] Y. Chen, "Soft handover issues in radio resource management for 3G WCDMA networks", PhD diss., 2003.

[16] N.B. Carvalho and J.C Pedro, "Multi-tone intermodulation distortion performance of 3rd order microwave circuits" 1999 IEEE MTT-S International Microwave Symposium Digest (Cat. No. 99CH36282). Vol. 2. IEEE, 1999. 